\title{
Robotic Assessment and Rehabilitation after Stroke
}

\author{
Sean P. Dukelow \\ Hotchkiss Brain Institute, Department of Clinical Neurosciences \\ $140329^{\text {th }}$ St. NW, Foothills Hospital, Calgary, Canada \\ spdukelo@ucalgary.ca
}

\section{Extended Abstract}

Stroke affects 60,000 Canadians annually. Stroke survivors are often left with a variety of impairments that can affect sensation, movement, visuospatial function, language and/or cognition. The nature and severity of these impairments cannot necessarily be predicted from routine clinical tests of the brain such as computed tomography or magnetic resonance imaging. Thus, clinicians rely on physical examination techniques and observer based ordinal scales to quantify the nature of post-stroke impairments. These bedside clinical assessments are used to diagnose impairments, prognosticate on recovery and serve as outcome measures for clinical trials. Unfortunately, many of these scales suffer from issues with floor or ceiling effects, reliability and precision. Our team has spent the last decade designing and validating a number of quantitative robotic assessments of post-stroke upper extremity function using a bimanual exoskeleton. We aim to help better understand the impairments that result from stroke and their effect on recovery.

The first part of this presentation will focus on the use of robotic technology for assessment of post-stroke upper extremity sensorimotor impairments. We collected data from over a hundred individuals with stroke and a similar number of controls have completed robotic and clinical assessments [1]. Stroke survivors completed 4 assessments during the first 6 months following stroke. To assess sensory function, in particular proprioception, we developed two robotic assessments. We examined position sense using a mirror matching task. In the tasks, subjects were required to mirror match the position where the stroke affected limb was moved by the robot. Forty-eight percent of individuals were impaired at 1 week post stroke versus $15 \%$ at 6 months as compared to normative values developed from controls. We also examined kinesthesia, the sense of limb motion, using another mirror matching task. Subjects were required to mirror match the speed, direction and amplitude of movements completely by the robotically moved stroke affected passive arm with their unaffected arm. Sixty-eight percent of individuals were impaired at 1 week post stroke and $23 \%$ at 6 months. We have also used voxelbased lesion symptom mapping, a technique that compares stroke lesion location to task performance, to determine brain area involved in both position matching and kinesthesia task performance.

These studies form the foundation for future work to be discussed in the second part of the talk. Appropriately identifying deficits allows the ability to plan targeted rehabilitation. We aim to examine the use of robotic technology as a rehabilitation tool. The first few weeks after stroke are a critical window for recovery where most of an individual's recovery occurs. Despite this, few studies have examined intensive rehabilitation in this time window. We are in the process of recruiting 110 individuals into a randomized controlled trial evaluating early (rehab beginning $\sim 7$ days poststroke) versus late (rehab beginning 21 days post-stroke) and more ( 2 hour/day) versus less (1 hour/day) robotic rehabilitation. This is in the hopes of determining whether the timing of robotic rehabilitation and/or dose has a significant impact on upper extremity outcomes at 6 months post-stroke.

\section{References}

[1] J. A. Semrau, T. M. Herter, S. H. Scott, and S. P. Dukelow, "Examining differences in patterns of sensory and motor recovery after stroke with robotics," Stroke, vol. 46, no. 12, pp. 3459-69, 2015. 